\title{
A Celtic Framework for Knots and Links
}

\author{
Jonathan L. Gross • Thomas W. Tucker
}

Received: 13 October 2009 / Revised: 11 January 2010 / Accepted: 21 February 2010 /

Published online: 17 March 2010

(C) Springer Science+Business Media, LLC 2010

\begin{abstract}
We describe a variant of a method used by modern graphic artists to design what are traditionally called Celtic knots, which are part of a larger family of designs called "mirror curves." It is easily proved that every such design specifies an alternating projection of a link. We use medial graphs and graph minors to prove, conversely, that every alternating projection of a link is topologically equivalent to some Celtic link, specifiable by this method. We view Celtic representations of knots as a framework for organizing the study of knots, rather like knot mosaics or braid representations. The formalism of Celtic design suggests some new geometric invariants of links and some new recursively specifiable sequences of links. It also leads us to explore new variations of problems regarding such sequences, including calculating formulae for infinite sequences of knot polynomials. This involves a confluence of ideas from knot theory, topological graph theory, and the theory of orthogonal graph drawings.
\end{abstract}

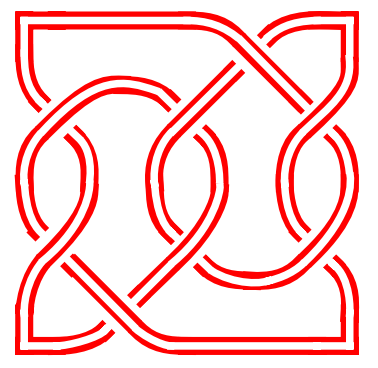

A Celtic knot

\footnotetext{
J.L. Gross (凶)

Department of Computer Science, Columbia University, New York, NY 10027, USA

e-mail: gross@cs.columbia.edu

url: http://www.cs.columbia.edu/ gross/
}

T.W. Tucker

Department of Mathematics, Colgate University, Hamilton, NY 13346, USA 


\section{Introduction}

Celtic knots are an ancient art form of continuing interest to modern graphic artists. Repetitive patterns and symmetries are among their geometric characteristics. In general, the art works of authentic Celtic origin that are called "Celtic knots" are topologically recognizable as alternating links. Various art experts have noted that similar figures (of a class called "mirror curves" [9]) that have occurred among Romans, Saxons, and Vikings, and also in some Islamic art and African art. Our exploration herein of Celtic knots blends knot theory, topological graph theory, and discrete geometry, with applications to computer graphics.

Our main concern is analyzing the topological properties of knots specified by Celtic designs. (For simplicity of exposition, we may sometimes say "knot" when our meaning is either a knot or a link.) We view Celtic representations of knots as a framework for organizing the study of knots, in the same spirit as, for example, knot mosaics [16], Gauss coding, or braid representations. Relevant background in knot theory is given, for example, by $[1,12,17]$, and [20].

Our topological graph theory terminology is consistent with [8] and [5]. We regard a normal projection of a link either as a graph or as a graph imbedded in the plane. Graph imbeddings are taken to be cellular and graphs to be connected, unless the alternative is declared or evident from context.

In computer-graphics research on Celtic knots by $[11,18]$ and others, the primary concern has been the creation of computer-assisted artwork that produces their classical geometric and stylistic features. Cyclic plain-weaving is a more general form of computer-assisted artwork, and, as observed by [2], the graphics it creates are alternating projections of links onto various surfaces in 3-space. From a topological perspective, Celtic knots and links are a special case of cyclic plain-weaving.

\section{Drawing a Celtic Knot}

To construct a barrier-free Celtic design, we begin with a $2 m \times 2 n$ rectangular array of grid-squares, where $m$ and $n$ are positive integers. Place a construction dot at each lattice-point $(x, y)$ such that $x+y$ is odd, where $0 \leq x \leq 2 n$ and $0 \leq y \leq 2 m$. Through each construction dot, draw two small line-segments that cross. If the $x$-coordinate is odd, the overcrossing is southwest to northeast; if even, the overcrossing is northwest to southeast. Also, the mirror image of a Celtic design (which switches overcrossings to undercrossings, and vice versa) is a Celtic design. Then join the ends of the segments to the ends of segments along the border or in diagonally adjacent grid-squares to form a rectangular plaitwork design, as shown in Fig. 1(a), which depicts a $4 \times 6$ barrier-free Celtic design.

In a barrier-free Celtic design, each construction dot lies at the center of a $1 \times 1$ subgrid, which contains one of the two types of crossings shown in Fig. 1(b), and is therefore called a crossing-subgrid. The operation of replacing a crossing-subgrid by one of the two types of $1 \times 1$ subgrids shown in Fig. 1(c) is called installing $a$ barrier, and those two subgrids are called barrier-subgrids. The heavier solid lines in the upper and lower subgrids are called a vertical barrier and a horizontal barrier, 
Fig. 1 Drawing a Celtic knot

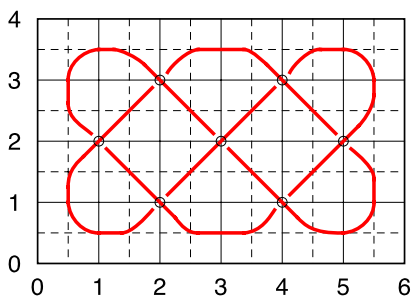

(a)
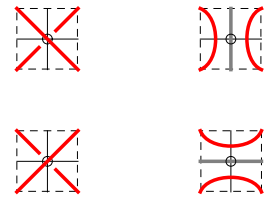

(b)

(c)

respectively. Any design resulting from the installation of barriers is called a Celtic design. The link within any Celtic design is called a Celtic knot.

M. Wallace [22] has posted a method in which the barriers are drawn first, based on publications of G. Bain [3] and I. Bain [4], intended for graphic artists, by which anyone capable of following directions can hand-draw Celtic knots, and which lends itself to implementation within a graphics system for creating computer-assisted art. Lomonaco and Kauffman [16] describe how a knot can be specified as a mosaic, in which the tiles contain crossings or the equivalent of barriers. Cromwell [6] constructs Celtic knots by replacing crossings in basic plaitwork. Our method and these three other methods all have much in common.

\section{Every Alternating Link is Celtic}

For the sake of completeness, we include a simple proof that every Celtic link, as we have defined it here, is alternating.

Theorem 3.1 Every Celtic diagram specifies an alternating link.

Proof It is easily proved that a barrier-free grid specifies an alternating link. Thus, before installing a barrier at a construction dot, the local pattern for an alternating link is as illustrated by Fig. 2 (left) or by a reflection of that figure. After installing the barrier, the local configuration is as in Fig. 2 (right) or its reflection. Thus, the link that results from splitting and reconnecting remains alternating.

One possible way to prove that every alternating link is topologically equivalent to some Celtic link is by induction on the number of crossings. The proof is reasonably straightforward, but involves numerous details and cases. Accordingly, we present a proof that draws on some basic concepts from topological graph theory, specifically medial graphs and graph minors.

\section{Medial Graphs and Inverse-Medial Graphs}

Given a cellular imbedding $\iota: G \rightarrow S$ of a graph in a closed surface, the medial graph $M_{\iota}$ (sometimes, just medial) is defined as follows:

- The vertices of $M_{\iota}$ are the barycenters of the edges of $G$. 
Fig. 2 Installing a barrier in an alternating link diagram

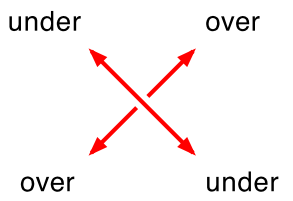

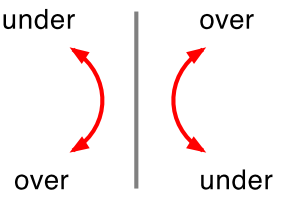

- For each face $f$ of the imbedding $\iota: G \rightarrow S$ and for each vertex $v$ of $G$ on $b d(f)$, install an edge joining the vertex of $M_{\iota}$ that immediately precedes $v$ in an fb-walk for $f$ to the vertex of $M_{\iota}$ that immediately follows $v$ on that fb-walk. (If the face $f$ is a monogon, then that edge is a self-loop.)

The imbedding $M_{\iota} \rightarrow S$ is called the medial imbedding for the imbedding $\iota: G \rightarrow S$, which we call, in turn, an inverse-medial of the imbedding $M_{\iota} \rightarrow S$.

Clearly, the medial imbedding is 4-regular. Moreover, since each face of the medial imbedding corresponds to either a vertex or a face of the original imbedding, the faces can be two-colored by the terms "vertex" or "face" so that each edge lies on one face of each color. These two properties characterize completely which 4-regular imbeddings are medial imbeddings.

Proposition 3.2 If the imbedding $M \rightarrow S$ is 4-regular with bipartite dual, then it has an inverse medial imbedding.

Proof Suppose the faces of the imbedding $M \rightarrow S$ are colored red and blue. Place a vertex at the center of each red face. For each vertex $v$ of $M$, draw an edge through $v$ between the centers of the two red faces incident to $v$. The resulting graph imbedding $G \rightarrow S$ has $M \rightarrow S$ as its medial. Note that if we had placed the centers in the blue faces instead, we would have the dual imbedding $G^{*} \rightarrow S$.

The following well-known fact identifies the characteristic of an imbedded 4-regular plane graph that permits it to have an inverse-medial graph.

Proposition 3.3 The dual of a 4-regular plane graph $G \rightarrow \mathbb{R}^{2}$ is bipartite.

Proof Each face of the dual graph is 4-sided. Since every cycle of a planar graph is made up of face-cycles, it follows that all cycles in the dual graph have even length, making the graph bipartite.

Theorem 3.4 Every 4-regular plane graph has two inverse-medial graphs.

Proof We observe that an imbedded graph and its dual have the same medial graph. Thus, this theorem follows from Propositions 3.3 and 3.2.

Inverse-Medial Graphs for Celtic Shadows

The image of a normal projection $\pi: L \rightarrow \mathbb{R}^{2}$ of a link is a 4-regular graph $G_{\pi}$ called the shadow of the link (e.g., see [17]); its vertices are the crossings, and its edges are the curves that join the crossings. Another consequence of Proposition 3.2 is as follows: 
Fig. 3 An inverse-medial for the shadow of the Celtic link $C K_{4}^{6}$

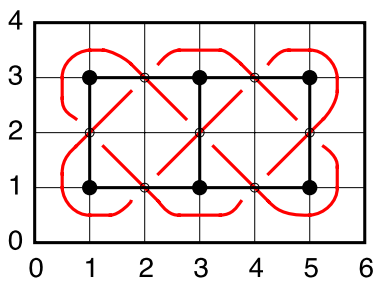

The link specified by the barrier-free $2 m \times 2 n$ Celtic diagram is denoted $C K_{2 m}^{2 n}$. In Fig. 3, we observe that a $1 \times 2$ orthogonal mesh (in black) is an inverse-medial graph for the shadow of the Celtic link $C K_{4}^{6}$.

Within any $2 m \times 2 c$ Celtic diagram the $(m-1) \times(n-1)$ orthogonal grid whose horizontals are on the lines $y=1,3,5, \ldots, 2 m-1$ and whose verticals are on the lines $x=1,3,5, \ldots, 2 n-1$ is called the inner grid of that diagram. As a graph, it is isomorphic to the Cartesian product $P_{m} \times P_{n}$ of the path graphs $P_{m}$ and $P_{n}$. We observe that every interior dot of the diagram lies at the midpoint of some edge of this grid. The outer grid is formed by the horizontals $y=0,2,4, \ldots, 2 m$ and the verticals $x=0,2,4, \ldots, 2 n$.

Proposition 3.5 The shadow of the Celtic link $C K_{2 m}^{2 n}$ has as one of its two inversemedial graphs the inner grid for the $2 m \times 2 n$ Celtic diagram.

Proof A formal approach might use an easy double induction on the numbers of rows and columns.

Remark In general, the other inverse-medial graph of the Celtic link $C K_{2 m}^{2 n}$ is obtained by contracting the border of the outer grid to a single vertex.

Clearly, every interior dot in a Celtic diagram is the midpoint of some edge of the inner grid, and every interior barrier in the diagram either coincides with an edge of the inner grid or lies orthogonal to the edge of that grid whose midpoint it contains.

Theorem 3.6 Given a Celtic diagram we can construct an inverse-medial graph for the shadow of the link it specifies as follows:

1. Start with the inner grid $M$.

2. Delete every edge of $M$ that meets a barrier orthogonally at its midpoint.

3. Contract every edge of $M$ that coincides with a barrier.

Proof Use induction on the number of barriers. This result follows from the given method for constructing the link specified by a Celtic diagram.

In view of Theorem 3.6, we can characterize each interior barrier in a Celtic diagram as a deletion barrier, if it meets an edge of the inner grid orthogonally, or as a contraction barrier, if it coincides with an edge of the inner grid. 
Fig. 4 (a) A Celtic link, its inner grid, and the barriers, (b) The inverse-medial of the shadow of that Celtic link

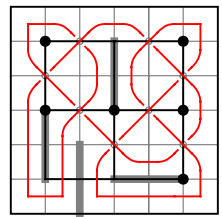

(a)

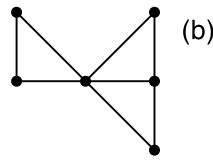

Example 3.1 We apply Theorem 3.6 to the Celtic link in Fig. 4. We delete the edge of the inner grid that is crossed by barriers, in the lower left corner of the diagram, and we contract the three edges of the mesh that coincide with barriers. The result is an inverse-medial for the shadow of the link, whose vertices are the black dots.

To obtain the other inverse-medial of the shadow of the given link, we would contract the edges of the outer grid that cross barriers and delete the edges that coincide with barriers. We would also contract the border of the diagram to a single vertex.

Corollary 3.7 One inverse-medial graph for the shadow of any link specified by a $2 m \times 2 n$ Celtic diagram is a minor of the graph $P_{m} \times P_{n}$, and the other is a minor of $P_{m+1} \times P_{n+1}$.

Proof This follows easily from Theorem 3.6.

Constructing a Celtic Diagram for an Alternating Link

By splitting a vertex of a graph, we mean inverting the operation of contracting an edge to that vertex.

Proposition 3.8 Let $\iota: G \rightarrow S$ be a graph imbedding such that some vertex of $G$ has degree greater than 3. Then it is possible to split that vertex so that the resulting graph is imbedded in $S$ and that the result of contracting the new edge is to restore the imbedding $\iota: G \rightarrow S$.

Proof This is a familiar fact that follows from elementary considerations in topological graph theory.

Proposition 3.9 Let $G$ be any planar graph with maximum degree at most 4 . Then $G$ is homeomorphic to a subgraph of some orthogonal grid.

Proof As explained, for instance, in Chap. 5 of [7] or in [21], every planar graph with maximum degree at most 4 has a subdivision that can be drawn as a subgraph of some orthogonal planar mesh.

The following Celtification algorithm constructs a Celtic diagram for any alternating link $L$ supplied as input.

1. Construct an inverse-medial graph $G$ for the shadow of the link $L$. 
2. Iteratively split vertices of $G$ as needed, so that every split graph is planar, and so that the final result $G^{\times}$has maximum degree at most 4 . After each such split at a vertex $v$, install a contraction barrier on the newly created edge-the edge with endpoint $v$.

3. Represent a subdivided copy of the planar graph $G^{\times}$as a subgraph of an orthogonal grid (as per Proposition 3.9), and enclose the grid by a border. Install a deletion barrier orthogonal to each edge of the grid that is not in the image of $G^{\times}$.

4. The resulting orthogonal grid with its contraction and deletion barriers is a Celtic design for the given link $L$.

Theorem 3.10 Let $L$ be an alternating link. Then there is a Celtic diagram that specifies $L$.

Proof We verify that each of the steps of the Celtification algorithm is feasible. Theorem 3.4 establishes that Step 1 is possible. Proposition 3.8 verifies the possibility of Step 2. Proposition 3.9 ensures that Step 3 is possible. Theorem 3.6 is the basis for Step 4.

\section{Celtistic Link Diagrams}

To generalize our scope, we define a Celtistic diagram to be otherwise like a Celtic diagram, except that we are permitted to specify at each dot whether the overcrossing is northwest to southeast or southwest to northeast. The Celtistic perspective on links simplifies the derivation of some kinds of general results and also facilitates the application of our methods of calculating knot polynomials for infinite sequences of knots and links.

\section{Theorem 3.11 Every link is Celtistic.}

Proof The proof that every alternating link is Celtic depends only on the shadow of the link, not on the overcrossings and undercrossings. Accordingly, we may apply the same argument here.

Corollary 3.12 Every 4-regular plane graph $G$ is the shadow of an alternating link.

Proof The straight-ahead walks (in a 4-regular graph, this means neither left nor right) in $G$ form the components of a link $L$. Of course, overcrossings and undercrossings could be assigned arbitrarily. By Theorem 3.11, the link $L$ could be specified by a Celtistic design. Changing the intersections so that they all follow the rules for a Celtic diagram produces a Celtic link $L^{\prime}$ whose shadow is the plane graph $G$.

Remark 1 An interpretation of Corollary 3.12 within Kauffman's terminology [12] is that every knot universe corresponds to some alternating knot. There are many different proofs of this widely known fact. 


\section{Some Geometric Invariants of Knots and Links}

Blending the theory of graph drawings (for an extensive survey, see [14]) with Theorems 3.10 and 3.11 suggests some interesting new geometric invariants of links. These four come immediately to mind:

- The Celtic area of a projection of a link $L$ is the minimum product $m n$ such that an equivalent projection $L$ is specifiable by a Celtistic diagram with $m$ rows and $n$ columns. The Celtic area of a link $L$, denoted $C_{A}(L)$, is the minimum Celtic area taken over all projections of $L$.

- The Celtic depth of a link projection is the minimum number $m$ such that a Celtistic diagram with $m$ rows specifies an equivalent projection. The Celtic depth of an alternating link $L$, denoted $C_{D}(L)$, is the minimum Celtic depth taken over all projections of $L$.

- The Celtic edge-length of a projection of a link $L$ is the minimum number of gridsquares traversed by the link in a Celtistic diagram for that projection. If a sublink of components of the link specified by the diagram splits off from the projection of $L$, then the edge-length of that sublink is not counted. The Celtic edge-length of a link, denoted $C_{L}(L)$, is the minimum Celtic edge-length taken over all projections. (This invariant is akin to what Kuriya [13] calls the mosaic number of a link.)

- The Celtic perimeter of a projection of a link $L$, denoted $C_{P}(L)$, is the minimum sum $4 m+4 n$, such that there is a $2 m \times 2 n$ Celtic diagram for $L$.

The following simple proposition is helpful in deriving values of these geometric invariants for specific links. Its proof is omitted. We use $\operatorname{cr}(\mathcal{D})$ for the number of crossings in a Celtistic diagram.

Proposition 4.1 Let $\mathcal{D}$ be a $2 m \times 2 n$ Celtistic diagram with $h$ horizontal barriers and $v$ vertical barriers.

- $\operatorname{cr}(\mathcal{D})+h+v=2 m n-m-n$.

- The Celtic depth of a non-trivial knot is at least 4.

- The Celtic area of a link with $x$ crossings is at least $2 x+4$.

Example 4.1 Using Proposition 4.1, we calculate some geometric invariants for the trefoil knot $3_{1}$ and for the figure-eight knot $4_{1}$ (Table 1).

We observe that the total curvature $\kappa(L)$ of a link in $\mathbb{R}^{3}$ (see [19]) can be bounded using Celtic invariants. For example, for the barrier-free Celtic knot in the $2 m \times 2 n$ grid, each corner supplies $\pi$ to the total curvature, and each of the $2(m-2)+2(n-2)$ curves at the sides adds $\pi / 2$. Thus the total curvature is at most $(m+n) \pi$. The

Table 1 Values of Celtic invariants for the trefoil knot and the figure-eight knot

\begin{tabular}{lllll}
\hline Knot & $C_{A}$ & $C_{D}$ & $C_{L}$ & $C_{P}$ \\
\hline 31 & 16 & 4 & 16 & 16 \\
$4_{1}$ & 24 & 4 & 24 & 20 \\
\hline
\end{tabular}


addition of each barrier increases the total curvature by at most $\pi$; however, adding barriers can also reduce the curvature. Since there are at most $2 m n-m-n$ barriers, we have the following upper bound:

Proposition 4.2 The total curvature of a link L satisfies the inequality

$$
\kappa(L) \leq C_{A}(L) \cdot \frac{\pi}{2}
$$

The total curvature invariant also provides upper bounds on other physical invariants of knots, such as thickness (see [15]). Accordingly, Celtic invariants can be related to those invariants as well.

\section{Knot Polynomials}

Celtic diagrams can be helpful when calculating knot polynomials for a recursively specifiable family of links. Indeed, they provide a way to identify families of links whose knot polynomials are amenable to recursive analysis. In this section, we derive recursions for the Alexander-Conway polynomials and the Kauffman bracket polynomials of the links specified by $4 \times 2 n$ barrier-free Celtic diagrams. The three smallest $4 \times 2 n$ barrier-free Celtic links are shown in Fig. 5 . We see that $C K_{4}^{6}$ is the knot 74 .

Alexander-Conway Polynomials

Definition The link diagrams $L$ and $L^{\prime}$ are equivalent link diagrams if one can be derived from the other by a sequence of Reidemeister moves.

Notation $L \sim L^{\prime}$.

We calculate the Alexander-Conway polynomial, denoted either by $\nabla_{K}$ or by $\nabla(K)$, of a knot (or link) $K$ by using the following three axioms.

Axiom 1. If $K \sim K^{\prime}$, then $\nabla_{K}=\nabla_{K^{\prime}}$.

Axiom 2. If $K \sim 0$, then $\nabla_{K}=1$.

Axiom 3. If $K, \bar{K}$, and $L$ are related as in Fig. 6, then $\nabla_{K}-\nabla_{K^{\prime}}=z \nabla_{L}$.

A link $L$ is said to be split if there is a 2-sphere in 3-space that does not intersect the link, such that at least one component of $L$ is on either side of the separation.

Fig. 5 Some small barrier-free $4 \times c$ Celtic knots
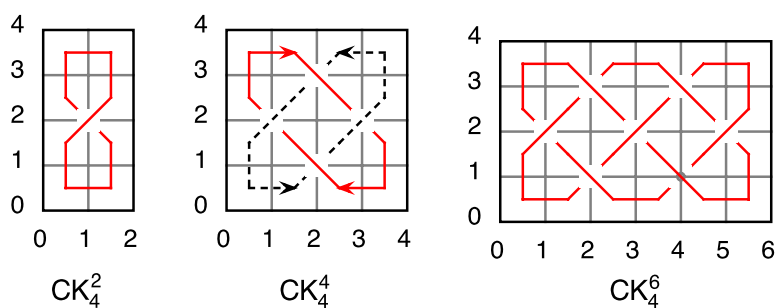
Fig. 6 Switch and elimination operations
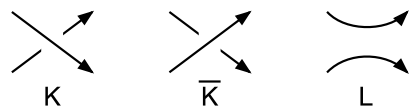

$\mathrm{L}$
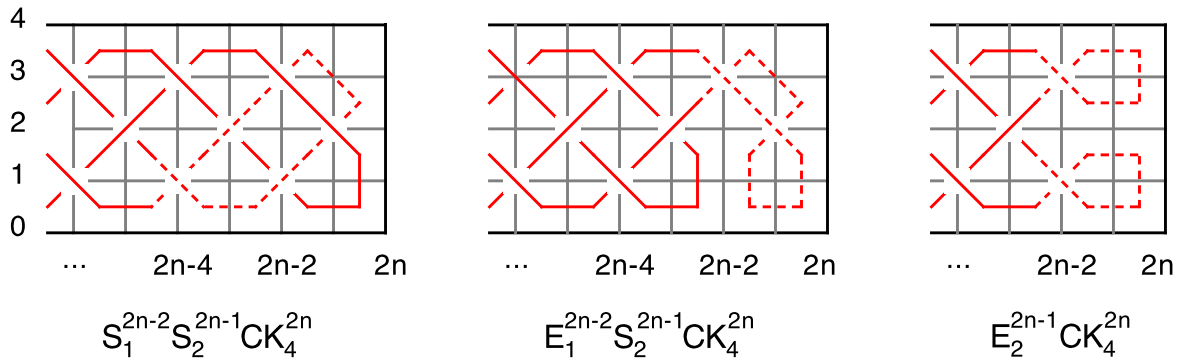

Fig. 7 Iterative operations on $C K_{4}^{2 n}$

Proposition 5.1 (a) The Alexander-Conway polynomial of the Hopf link, with either mix of component orientations, is $z$. (b) The Alexander-Conway polynomial of a split link is 0 . (c) The Alexander-Conway polynomial of a trefoil knot is $1+z^{2}$.

Proof These polynomials are well known.

Notation The notation $S_{r}^{c}$ means switch the crossing of a Celtic link $K$ at row $r$, column $c$. The notation $E_{r}^{c}$ means eliminate the crossing at row $r$, column $c$.

Remark The orientations of the components of the link matter quite a lot, in particular, when calculating the Alexander-Conway polynomial or the genus of a link.

Lemma 5.2 The following three relations hold for operations on Celtic links.

$$
\begin{aligned}
S_{1}^{2 n-2} S_{2}^{2 n-1} C K_{4}^{2 n} & \sim C K_{4}^{2 n-4} \\
E_{1}^{2 n-2} S_{2}^{2 n-1} C K_{4}^{2 n} & \sim C K_{4}^{2 n-2} \\
E_{2}^{2 n-1} C K_{4}^{2 n} & \sim C K_{4}^{2 n-2}
\end{aligned}
$$

Proof These relations follow from the diagrams in Fig. 7. Retracting the dashed parts of the links corresponds to Reidemeister moves.

Theorem 5.3 The coefficients of the Alexander-Conway polynomial for the barrierfree link sequence $C K_{4}^{2}, C K_{4}^{4}, C K_{4}^{6}, \ldots$ are given by this formula:

$$
\nabla\left(C K_{4}^{2 n}\right)=\sum_{k=0}^{n-1} b_{k}^{2 n} z^{k} \quad \text { where } b_{k}^{2 n}= \begin{cases}0 & \text { if } k \equiv n \bmod 2 \\
\left(\begin{array}{c}
(n+k-1) / 2 \\
k
\end{array}\right) 2^{k} & \text { otherwise }\end{cases}
$$


Proof We first establish this recursion:

$$
\begin{aligned}
\nabla\left(C K_{4}^{0}\right) & =0 \\
\nabla\left(C K_{4}^{2}\right) & =1 \\
\nabla\left(C K_{4}^{2 n}\right) & =\nabla\left(C K_{4}^{2 n-4}\right)+2 z \nabla\left(C K_{4}^{2 n-2}\right) \quad \text { for } n \geq 2
\end{aligned}
$$

Equation (5.4) is a normalization constant. Since $C K_{4}^{2}$ is an unknot, (5.5) follows from Axiom 2. We now verify (5.6).

$$
\begin{aligned}
\nabla\left(C K_{4}^{2 n}\right)= & \nabla\left(S_{2}^{2 n-1} C K_{4}^{2 n}\right)+z \nabla\left(E_{2}^{2 n-1} C K_{4}^{2 n}\right) \quad \text { (Axiom 3) } \\
= & {\left[\nabla\left(S_{1}^{2 n-2} S_{2}^{2 n-1} C K_{4}^{2 n}\right)+z \nabla\left(E_{1}^{2 n-2} S_{2}^{2 n-1} C K_{4}^{2 n}\right)\right] } \\
& +z \nabla\left(E_{2}^{2 n-1} C K_{4}^{2 n}\right) \quad(\text { Axiom 3) } \\
= & \nabla\left(C K_{4}^{2 n-4}\right)+2 z \nabla\left(C K_{4}^{2 n-2}\right) \quad(\text { Lemma 5.2) }
\end{aligned}
$$

To obtain $b_{k}^{2 n}$ as the coefficient of $t^{2 n} u^{k}$, the generating function is

$$
\frac{t^{2}}{1-t^{2}\left(t^{2}+2 u\right)}
$$

The conclusion follows.

Example 5.1 Applying Theorem 5.3 gives these Alexander-Conway polynomials:

$$
\begin{aligned}
& \nabla\left(C K_{4}^{4}\right)=2 z \\
& \nabla\left(C K_{4}^{6}\right)=1+4 z^{2}, \quad \text { and } \\
& \nabla\left(C K_{4}^{8}\right)=4 z+8 z^{3}
\end{aligned}
$$

Kauffman Bracket Polynomials

Kauffman's bracket polynomial is defined by three axioms:

Axiom 1. $\langle\bigcirc\rangle=1$

Axiom $2 \mathrm{u} .\langle Y\rangle=A\langle)(\rangle+A^{-1}\left\langle\leftrightharpoons^{\smile}\langle/\rangle\right.$-overcross

Axiom 2d. $\langle\backslash\rangle=A\langle\leftrightharpoons\rangle+A^{-1}\langle)(\rangle\langle\rangle$-overcross

Axiom 3. $\langle L \cup \bigcirc\rangle=\left(-A^{2}-A^{-2}\right)\langle L\rangle$

Calculating the Jones polynomial of a link is known to be $\# P$-hard [10], and the coefficients of a Jones polynomial can be calculated by making a substitution into a bracket polynomial. Accordingly, the general problem calculating the bracket polynomial of links is regarded as computationally intractable. Nonetheless, there remains the possibility of calculating bracket polynomials for an infinite sequence of links, as we now illustrate. 
Fig. 8 Celtic diagrams for bracket polynomial relations

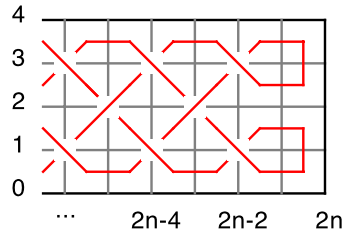

(a) $\mathrm{H}_{2}^{2 \mathrm{n}-1} \mathrm{CK}_{4}^{2 \mathrm{n}}$

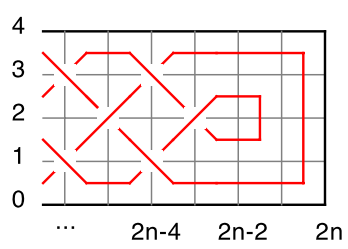

(c) $\mathrm{H}_{3}^{2 n-2} \mathrm{H}_{1}^{2 n-2} \mathrm{~V}_{2}^{2 n-1} \mathrm{CK}_{4}^{2 n}$

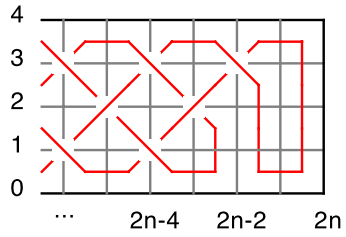

(b) $\mathrm{V}_{1}^{2 \mathrm{n}-2} \mathrm{~V}_{2}^{2 \mathrm{n}-1} \mathrm{CK}_{4}^{2 \mathrm{n}}$

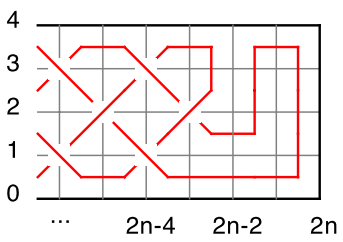

(d) $\mathrm{V}_{3}^{2 n-2} \mathrm{H}_{1}^{2 n-2} \mathrm{~V}_{2}^{2 n-1} \mathrm{CK}_{4}^{2 n}$

Notation The notation $H_{r}^{c}$ means replace the crossing of a Celtic link $K$ at row $r$, column $c$ by a horizontal pair. The notation $V_{r}^{c}$ means replace the crossing at row $r$, column $c$ by a vertical pair.

Lemma 5.4 The following four relations hold for bracket polynomials:

$$
\begin{aligned}
\left\langle H_{2}^{2 n-1} C K_{4}^{2 n}\right\rangle & =A^{-6}\left\langle C K_{4}^{2 n-2}\right\rangle \\
\left\langle V_{1}^{2 n-2} V_{2}^{2 n-1} C K_{4}^{2 n}\right\rangle & =-A^{-3}\left\langle C K_{4}^{2 n-2}\right\rangle \\
\left\langle H_{3}^{2 n-2} H_{1}^{2 n-2} V_{2}^{2 n-1} C K_{4}^{2 n}\right\rangle & =-A^{3}\left\langle V_{2}^{2 n-3} C K_{4}^{2 n-2}\right\rangle \\
\left\langle V_{3}^{2 n-2} H_{1}^{2 n-2} V_{2}^{2 n-1} C K_{4}^{2 n}\right\rangle & =\left\langle C K_{4}^{2 n-2}\right\rangle
\end{aligned}
$$

Proof Equations (5.7), (5.8), (5.9), and (5.10), follow from the diagrams (a), (b), (c), and (d), respectively in Fig. 8.

Remark Equations (5.7), (5.8), and (5.9) reflect the fact that the bracket polynomial is not preserved by the first Reidemeister move. Indeed, the first Reidemeister move changes the bracket polynomial by $A^{3}$ or $A^{-3}$, depending on the direction of the twisting or untwisting.

Theorem 5.5 The bracket polynomial for the barrier-free link sequence $C K_{4}^{0}$, $C K_{4}^{2}, C K_{4}^{4}, C K_{4}^{6}, \ldots$ is given by the following recursion:

$$
\begin{aligned}
\left\langle C K_{4}^{0}\right\rangle & =0 \\
\left\langle V_{2}^{1} C K_{4}^{2}\right\rangle & =1 \\
\left\langle C K_{4}^{2}\right\rangle & =-A^{-3} \\
\left\langle V_{2}^{2 n-1} C K_{4}^{2 n}\right\rangle & =\left(1-A^{-4}\right)\left\langle C K_{4}^{2 n-2}\right\rangle-A^{5}\left\langle V_{2}^{2 n-3} C K_{4}^{2 n-2}\right\rangle \quad \text { for } n \geq 2 \\
\left\langle C K_{4}^{2 n}\right\rangle & =A\left\langle V_{2}^{2 n-1} C K_{4}^{2 n}\right\rangle+A^{-7}\left\langle C K_{4}^{2 n-2}\right\rangle \text { for } n \geq 2
\end{aligned}
$$


Proof Equation (5.11) is a normalization constant, and (5.12) and (5.13) are easily verified from fundamentals. We now confirm (5.14) and (5.15).

$$
\begin{aligned}
&\left\langle V_{2}^{2 n-1} C K_{4}^{2 n}\right\rangle= A\left\langle H_{1}^{2 n-2} V_{2}^{2 n-1} C K_{4}^{2 n}\right\rangle \\
&+A^{-1}\left\langle V_{1}^{2 n-2} V_{2}^{2 n-1} C K_{4}^{2 n}\right\rangle \quad \text { (by Ax. 2d) } \\
&= A\left\langle H_{1}^{2 n-2} V_{2}^{2 n-1} C K_{4}^{2 n}\right\rangle \\
&+A^{-1}\left(-A^{-3}\right)\left\langle C K_{4}^{2 n-2}\right\rangle \quad(\text { by }(5.8)) \\
&=-A^{-4}\left\langle C K_{4}^{2 n-2}\right\rangle+A\left[A\left\langle H_{3}^{2 n-2} H_{1}^{2 n-2} V_{2}^{2 n-1} C K_{4}^{2 n}\right\rangle\right. \\
&\left.+A^{-1}\left\langle V_{3}^{2 n-2} H_{1}^{2 n-2} V_{2}^{2 n-1} C K_{4}^{2 n}\right\rangle\right] \quad \text { (by Ax. 2d) } \\
&=-A^{-4}\left\langle C K_{4}^{2 n-2}\right\rangle+A^{2}\left(-A^{3}\right)\left\langle V_{2}^{2 n-3} C K_{4}^{2 n-2}\right\rangle \\
&+A \cdot A^{-1}\left\langle C K_{4}^{2 n-2}\right\rangle \quad(\text { by }(5.9,5.10)) \\
&=\left(1-A^{-4}\right)\left\langle C K_{4}^{2 n-2}\right\rangle-A^{5}\left\langle V_{2}^{2 n-3} C K_{4}^{2 n-2}\right\rangle \\
&\left\langle C K_{4}^{2 n}\right\rangle=A\left\langle V_{2}^{2 n-1} C K_{4}^{2 n}\right\rangle+A^{-1}\left\langle H_{2}^{2 n-1} C K_{4}^{2 n}\right\rangle \quad \text { (by Ax. 2u) } \\
&=A\left\langle V_{2}^{2 n-1} C K_{4}^{2 n}\right\rangle+A^{-1} A^{-6}\left\langle C K_{4}^{2 n-2}\right\rangle \quad \text { (by (5.7)) } \\
&=A\left\langle V_{2}^{2 n-1} C K_{4}^{2 n}\right\rangle+A^{-7}\left\langle C K_{4}^{2 n-2}\right\rangle
\end{aligned}
$$

Example 5.2 Applying Theorem 5.5 gives these bracket polynomials:

$$
\begin{aligned}
\left\langle V_{2}^{3} C K_{4}^{4}\right\rangle= & A^{-7}-A^{-3}-A^{5} \quad \text { knot } 3_{1} \\
\left\langle C K_{4}^{4}\right\rangle= & -A^{-10}+A^{-6}-A^{-2}-A^{6} \quad \text { link } 4_{1}^{2} \\
\left\langle V_{2}^{5} C K_{4}^{6}\right\rangle= & A^{-14}-2 A^{-10}+2 A^{-6}-2 A^{-2} \\
& +2 A^{2}-A^{6}+A^{10} \text { knot } 62 \\
\left\langle C K_{4}^{6}\right\rangle= & -A^{-17}+2 A^{-13}-3 A^{-9}+2 A^{-5} \\
& -3 A^{-1}+2 A^{3}-A^{7}+A^{11} \text { knot } 7_{4}
\end{aligned}
$$

Remark Whereas the time to calculate $\left\langle C K_{2 n}^{4}\right\rangle$ by the usual skein relations is exponential in $n$, we observe that each iteration of the recursions (5.14) and (5.15) increases the span of the bracket polynomial by at most 12 . Thus, the time needed to calculate $\left\langle C K_{2 n}^{4}\right\rangle$ by applying these recursions is quadratic in $n$.

\section{Conclusions}

Celtic design can be used to specify any alternating link and that Celtistic design can be used to specify any link. We have seen that Celtic representation suggests some 
new geometric invariants, which can yield information about some well-established knot invariants. It can also be used to calculate knot polynomials for infinite families of knots and links. Moreover, the computation time for bracket polynomials (or Jones polynomials) by the methods given here is quadratic in the number of crossings, in contrast to the standard exponential-time skein-based recursive algorithm.

\section{References}

1. Adams, C.C.: The Knot Book. Am. Math. Soc., Providence (2004). Original edn. Freeman, 1994

2. Akleman, E., Chen, J., Xing, Q., Gross, J.L.: Cyclic plain-weaving with extended graph rotation systems. ACM Trans. Graph. 28 (2009), Article \#78. Also SIGGRAPH 2009, 100-108

3. Bain, G.: Celtic Art: The Methods of Construction. Dover, New York (1973). Original edn. William Maclellan, Ltd, Glasgow, 1951

4. Bain, I.: Celtic Knotwork. Sterling Publishing Co., New York (1997). Original edn. Constable, Great Britain, 1986

5. Beineke, L.W., Wilson, R.J., Gross, J.L., Tucker, T.W. (eds.): Topics in Topological Graph Theory. Cambridge University Press, Cambridge (2009)

6. Cromwell, P.R.: The distribution of knot types in Celtic interlaced ornament. J. Math. Arts 2, 61-68 (2008)

7. DiBattista, G., Eades, P., Tamassia, R., Tollis, I.G.: Graph Drawing: Algorithms for the Visualization of Graphs. Prentice-Hall, New York (1999)

8. Gross, J.L., Tucker, T.W.: Topological Graph Theory. Dover, New York (2001). Original edn. Wiley, 1987

9. Jablan, S.: Mirror curves. Internet website contribution, http://modularity.tripod.com/mirr.htm

10. Jaeger, F., Vertigan, D.L., Welsh, D.J.A.: On the computational complexity of the Jones and Tutte polynomials. Math. Proc. Camb. Philos. Soc. 108, 35-53 (1990)

11. Kaplan, M., Cohen, E.: Computer generated Celtic design. In: Proc. 14th Eurographics Symposium on Rendering, pp. 9-16 (2003)

12. Kauffman, L.H.: Formal Knot Theory. Dover, New York (1983). Original edn. Princeton University Press, 1983

13. Kuriya, T.: On a Lomonaco-Kauffman conjecture, arXiv:0811.0710

14. Liotta, G., Tamassia, R.: Drawings of graphs. In: Gross, J.L., Yellen, J. (eds.) Handbook of Graph Theory. CRC Press, Boca Raton (2004), Chap. 10.3

15. Litherland, R.A., Simon, J., Durumeric, O., Rawdon, E.: Thickness of knots. Topol. Appl. 91, 233244 (1999)

16. Lomonaco, S.J., Kauffman, L.H.: Quantum knots and mosaics, J. Quantum Inf. Process. 7, 85-115 (2008). arXiv:0805.0339

17. Manturov, V.: Knot Theory. CRC Press, Boca Raton (2004)

18. Mercat, C.: Les entrelacs des enluminure celtes. Dossier Pour La Science 15 (January, 2001)

19. Milnor, J.W.: On the total curvature of knots. Ann. Math. 52, 248-257 (1950)

20. Murasugi, K.: Knot Theory and Its Applications. Birkhäuser, Basel (1998). Original edn., 1996

21. Storer, J.A.: The node cost measure for embedding graphs on the planar grid. In: Proceedings of the 12th Annual ACM Symposium on Theory of Computing, pp. 201-210 (1980)

22. Wallace, M.: Constructing a Celtic knot. Internet website contribution, http://www.wallace.net/knots/ howto/ 